In carrying out the general rule, that mercury should be given as long as induration lasts, the surgeon must not forget, that in old-standing cases, the mass may consist of something more than specific induration, and may be made up of organized tissue, which it is in vain to think of removing by mercury, as is so ably stated by M. Ricord and Wallace.* I have myself seen persons bearing traces of induration for two years after a course of mercury has been left off, and yet no secondary symptoms follow; but in these instances it has been impossible, from the situation of the hardened mass, to apply compression. I now generally find these "remains" less common than formerly: the surgeon, however, must be prepared to leave them occasionally, particularly when he thinks he has given norcury enough, or the constitution of his patient will not allow him to carry it further.

In concluding these remarks, I have to apologize to my readers for dwelling so long on the subject of indurated chancre; but let it be remembered that induration is the keystone to the arch of syphilis, and that I have attempted to collect together all the available knowledge which science possesses, and have added my onn observations. Much, however, still remains un. known; and $I$ hope others will undertake the investigation of this scientific question, particularly the influence of climate and treatment on the hardening of sores.

Queen-Ann-street, Cavendish-square.

\section{ON A RARE FORM OF DISLOCATION OF THE ASTRAGALUS.}

BY J. H. MACDONNELL, M.D., Dublin.

In Twe LaNCEr for January $30 \mathrm{th}$, (p. 133,) there is a report from the London Itospital, of a highly interesting and rare case of dislocation of the astragalus, in which the head of this bone was forcibly driven inwards, and slightly upwards, from its connexion with the navicular bone. Mr Adams remarks, that he knows of no recorded case similar to his; and I am satisfied, after a careful search for such cases, that they are of extreme rareness. No such case is mentioned, as of ordinary occurrence, by Sir A. Cooper, Boyer, Chelius, or Gibson; from which I infer that the dislocation is not in the list of those recognised by the surgeons of Englan l, France, Germany, or the United States.

I have, however, met with a case very nearly resembling this, which occurred in the person of Mr. Carmichael, the distinguished surgeon of the Richmond Hospital, Dublin, in 1839. The dislocation was occasioned by the sudden fall of Mr. Carmichael's horse, while he was moving at a brisk trot. Mr. Carmichael's weight was thrown, with great violence, on the anterior part of the right foot, which alone came to the ground. About lialf an hour after the accident, he was seen by Dr. IIutton and myself, when we observed the following deformity. The toes were turned outwards, the inner edge of the foot forming an angle of about thirty degrees, with its natural direction; the sole was slightly turned outwards, and the outer edge slightly elevated. The concavity of the tendoAchillis posteriorly was increased, and the heel lengthened. On grasping the soft parts between the tendo-Achillis and tibia, we found the distance between them much greater than in the other leg. The absence of the hard projection, which would have been formed by the upper part of the astragalus, had it passed backwards with the rest of the tarsus, was evident. Each malleolus was perfectly defined: below and before the inmer, there was a hard prominence, over which the skin was tense, formed by the inner surface of the astragalus, brought into relief by the dislocation and the slight eversion of the sole of the foot. Much the most striking

* "We will lay down as a general rule, that the surgeon should continue the mercury as long as induration remains. To remove a moderate-sized
induration, treatment should be persisted in for six months, ptovided the induration, treatment should be persisted in for six months, ptovided the
mineral produces no ill consequences. But (adds he) a six months' treatmineral produces no ill consequences. But (adds he) a six monthis' treat-
ment is no slight matter, and you should not prescribe it except when you ment is no slight matter, and you should not prescribe it cxcept when you are unable to do otherwise; and it would be wcll for our patient if this
could be aroided." Aqain, lower down, he says : "We ought to continue mercury until the entire disappearance of the induration around the chancre. But here springs up a difficulty. Is this induration always easy chancre. But here springs up a diffeulty. Is this induration always easy destroyed, there remains sometimes du tissu inodulaire, des tissus de cica. trice, des nodules de tissu fibro-cartilaginerse, which may impose upon us as the remains of induration. Mistrust renerally an induration which resists, during six or eight months, a well-regulated mercurial treatment." resicord Garcte dus

Ricord, Gazetie de's Hôpitau.

to is, howerer, to be observed, that it will not be always in our power to disperse indurations of this kind; for it sometimes happens that a state mercury and tightening will continue long after the period at which of the parts which ho for life."-Walluce, loc. cit., p. 312 . feature of the deformity consisted in a prominence on the dorsum of the foot. Immediately in front of the tibia, this prominence presented a flat surface, broad, from the tibia forwards, about a finger's breadth, from which there was an abrupt descent to the anterior part of the tarsus. Over this projection, caused by the head of the astragalus thrown on the upper surface of the scaphoid and cuneiform bones, the integuments were so tense, that it was evident a very small additional force would have driven the astragalus through them. Lastly, on taking the distance from the point of the internal malleolus to the extremity of the great toe with a tape measure, I found it to be almost an inch less than the distance between the same points in the left foot. We could detect no fracture. The foot could be flexed and extended; but, as any movement occasioned great pain, we did not ascertain to what extent flexion and extension could be carried. There was no obscurity about these deviations from the natural form. There had not been time for in Hammatory tumefaction to arise, and no effusion of blood had taken place.

The conclusion we came to was, that the violence had re. sulted in tearing the astragalus from its bed, on the os calcis, the inferior calcanco-scaphoid ligament, and scaphoid bone, and throwing it ca the upper surface of the scaphoid and cuneiform bones.

Unlike the case recorded in THe LANCEx, we experienced considerable difficulty in the reduction of the dislocation. We were under the necessity of having recourse to pulleys, and even with their assistance, the reduction was difficult.

A detail of the manner in which the luxation was produced, and of the means used in the reduction, may be seen in the fourteenth volume (p. 235) of the Dublin Joneral of Micdical Science.

Excent in the precise direction of the luxation, the London Hospital case was, I think, very similar to this. The reporter (Mr. Adams) would, I am sure, gratify the profession, if he would favour then with a more minute detail than he has given of his very interesting case.

Dublin, Feb., 18 s.

\section{THE LAW OF THE MORPHOLOGY OR META. MORPHOSIS OF THE TEXTURES OF THE HUMAN BODY.}

\section{By WILLIAM ADDISON, M.D., F.R.S., Malvern.}

$$
\text { II.- }
$$

It may with great certainty be affirmed, that the blood contains the elements from which, in animal structures, the solid textures and the secretions are produced. Blood con. sists of a limpid find, holding in suspension a multitude of colls of two hinds; the one red, the other white or colourless; it may therefore with propriety be termed a cellular or corpuscular fluid. On its first discharge from the living vessels, blood is thin-that is to say, it drops like water, but it speedily becomes viscous or stringy, and then coagulates into a sof solid, which ultimately separates into two parts-a red clot, and a yellow, fuid serum. But blood, before it coagulates, frequently separates into two fluid portions; the uppermost colourless, and termed plasma, lymph, or liquor sanguinis, the lower red: both these portions coagulate, so that the solid clot is in part colourless and in part red. The colourless part of the clot, which is uppermost, and formed by the coagulation of the plasma or lymph, is a coherent and elastic fibro-cellular texture, ordinarily termed the buffy coat; it is usually depressed in the centre, with thin, fibrous edges, and there is frequently a viscous, colourless, gelatinous mucus in the cup-like depression, that may be drawn out in strings like ordinary mucus.

The microscopic appearances in a film of blood not disposed to exhibit a colourless layer of lymph or plasma, are very different from those of a film of blood that is disposed to do so. In blood not disposed to exhibit a buffy coat upon the clot, we see with the microscope numerous red corpuscles or cells, slightly cohering in strings and rolls, and a few colourless ones floating in a clear, limpid fluid. In blood that is disposed to have a buffy coat upon the clot, we see a much larger proportion of colourless cells, and multitudes of minute molecules floating with the red cells in the clear and limpid fluid; the red cells adhere more firmly to each other, and ara congregated in larger and more irregular masses; and in the fluid, a network or tissue of interlaced filaments or fibrils is shortly observed to form. If a film of the colourless fluid, plasma, or lympl, be separately examined with the micru- 